complete guidance, but this does not prevent him from writing a brilliant account in which the central ideas - and equations - are presented in the midst of much on the personal side.

This is the point where the split of physics into many branches began. The narrative narrows, but an enormous amount of material nevertheless remains to be covered in the closing five chapters of the "History". It could not have been done with greater artistry. The interwoven strands tell us about quantum electrodynamics and the positron, about the continuing mystery of $\beta$-decay, leading up to the neutrino, about the merging concepts of fields and of particles that are born and die, and how a deepening understanding of symmetries has proved to be the most reliable guide to progress. In all this Pauli and Dirac, among others, are most vividly portrayed.

Inevitably, much prominence has to be given to the great shadow cast on physics by the knowledge that the theoretical structures used at the time were quite unsound mathematically, and that the necessarily approximate calculations were completely unreliable. The "History" was bound to end on such an unhappy note, but Pais has waited to its last chapter to pick up a final, more optimistic theme, going back to 1932 and the discovery of the neutron. At last there was then a convincing picture of nuclear structure to develop, into which Fermi managed to inject an at least provisionally acceptable description of $\beta$-decay. It was from there that the search for an account of nuclear forces started and, to close the story, we hear how Anderson's discovery of the muon and Yukawa's theory of the pion (as they were later to be called) seemed to open new vistas. It is left to the subsequent "Memoir" to tell us how wrong our ideas at that time were. I say "our" because this last episode of the "History" is the first of what might have been "Memoir" for me. I can vouch for the complete authenticity of the story.

The "Memoir" starts with the entirely new situation in physics after the Second World War. Many techniques, many young trained minds and large resources become available, in particular in the United States. Having survived years of danger and tragedy in his native Netherlands, Pais is there to take part in the explosive growth of his subject. The "Memoir" is the story of his life in physics and with physicists, in the centre of unprecedented developments in both experiment and theory. These Pais manages to absorb and to reproduce for the reader, still vividly but inevitably in a more technical manner than many readers (including trained physicists not on the inwardbound track) will only be able to follow in detail thanks to the extensive references.

There are just four chapters in the
"Memoir" and it would be difficult to enumerate everything in them. The "strand" structure is as before, the first three chapters telling the story up to 1960. We learn of the transformation of theoretical thinking with the arrival of the idea of renormalization and its success in explaining observed facts in electrodynamics; of the impact of the big machines and of their characteristics; of the discovery of many new particles and of the new symmetries that help to put some order into the picture; of the shock when long-cherished old symmetries turn out to be violated; and of many other avenues that are explored, some fruitlessly.

How Pais manages to fit in an account of the still-more-turbulent developments from 1960 onwards into one chapter of a

\section{Membrane steps}

\section{Miles Houslay}

Reconstitutions of Transporters, Receptors, and Pathological States. By Efraim Racker. Academic: 1985. Pp. 271. Hbk $\$ 28, £ 24.50 ; p b k \$ 12.95, £ 12$.

EFraIm Racker is one of the founding fathers of membrane reconstitution, and the prospect of reading his book on the subject rather intrigued me. What would be his perception of the past, present and future of membrane biology? In the preface Racker says that the book is based on a lecture series he was used to giving, which sounds promising. Each of the lectures ( 12 in all) is dedicated to putting over a series of 34 "lessons" varying from good general advice to homespun philosophy. A certain amount of sound advice is indeed to be welcomed by the aspiring "reconstitutor", as taking membranes apart and putting them back together is like gardening, and requires the equivalent of green fingers and a certain feel for the subject.

Lectures 1 and 2 have the central theme of "how to solubilize the protein that you've chosen". Here Racker makes the important point that what works for one protein may well not succeed for another (that is, you often have "to suck it and see"), and lists a variety of parameters of which one has to be aware. He makes it abundantly clear that there is no selfevident set of rules to follow: there are too many parameters and we have too little basic appreciation of how individual membrane proteins are glued into bilayers.

However, having said all this, the reader is then offered little or no constructive help. We are advised to take account of "critical micellar concentrations" and hydrophil-lipophil balances, but without even being told what they are, never mind how such values may be of use to tease mere 70 pages (with eight pages of references to end with) is almost miraculous. $\mathrm{He}$ shoots the discoveries and ideas at the reader with machine-gun rapidity - but it is still the same author writing, keeping the human side up with the technical.

If I who have lived through the whole period of the "Memoir", but with far less first-hand experience of the great events, ever attempt to get my bearings on these modern things, from quarks to the $\mathrm{Z}$ particle, with the description of things like colliders thrown in. I know I shall turn to Pais, both his narrative and his references. It is an inimitable work.

Nicholas Kemmer, 35 Salisbury Road, Edinburgh EH16 5AA, UK, is Emeritus Professor of Mathematical Physics at the University of Edinburgh.

proteins out of their membrane home. Purification of membrane proteins, which is after all the main goal of many people interested in this field, gets a desultory page without any references to different ways of coping with this key problem. A series of general methods for reconstitution then follows, with lists of some reconstituted systems and their "activities". These lists (again innocent of references) are of little use except to give a broad view of the variety of systems that the author has got hold of and stuck back into liposomes, though the accompanying, anecdotal text does convey some idea of the principles involved.

The succeeding chapters all suffer from similar drawbacks. The section headings identify important points that should be investigated or considered, but the monologue that follows is superficial and jumps haphazardly from one system to another. One can have a degree of sympathy with such an approach in the introduction, but after three chapters one finds oneself desperately hoping to come across a logical presentation of hard facts. As it is, over the next seven chapters we are led a merry dance around a variety of membrane systems. The penultimate lecture is about cancer; it raises a few interesting points about glycolysis but is largely an indulgence, as is the final lecture centring on the author's current predilections.

I am afraid to say that after having had such high hopes for this book I found it difficult to read to the end; it lacks both substance and cohesion. Further, although Racker apologizes for all those people he has not mentioned, the number of self and selective citations in places leads to history being re-written. I would not recommend the book to students, but lecturers running courses on membranes, or sociologists of science, might find it of some interest.

Miles Houslay is Gardiner Professor in the Department of Biochemistry, University of Glasgow, Glasgow G12 $8 Q Q$, UK. 